\title{
Discours
}

Revue de linguistique, psycholinguistique et

informatique. A journal of linguistics, psycholinguistics and computational linguistics

14 | 2014

Varia

\section{Étude comparée de l'usage parenthétique des verbes épistémiques dans trois langues romanes}

\section{Gerda Haßler}

\section{(2) OpenEdition}

Journals

Édition électronique

URL : http://journals.openedition.org/discours/8888

DOI : $10.4000 /$ discours. 8888

ISSN : 1963-1723

Éditeur :

Laboratoire LATTICE, Presses universitaires de Caen

\section{Référence électronique}

Gerda Haßler, «Étude comparée de l'usage parenthétique des verbes épistémiques dans trois langues romanes », Discours [En ligne], 14 | 2014, mis en ligne le 16 septembre 2014, consulté le 02 mai 2019. URL : http://journals.openedition.org/discours/8888 ; DOI : 10.4000/discours.8888

\section{(c) (i) (9) $९$}

Discours est mis à disposition selon les termes de la licence Creative Commons Attribution - Pas d'Utilisation Commerciale - Pas de Modification 4.0 International. 

Revue de linguistique, psycholinguistique et informatique

\section{Étude comparée de l'usage parenthétique} des verbes épistémiques dans trois langues romanes

Gerda Haßler

Institut de romanistique

Université de Potsdam

Gerda Haßler, «Étude comparée de l'usage parenthétique des verbes épistémiques dans trois langues romanes», Discours [En ligne], 14 | 2014, mis en ligne le 16 septembre 2014.

URL: http://discours.revues.org/8888

Titre du numéro: Varia

Coordination: Laure Sarda et Denis Vigier 



\title{
Étude comparée de l'usage parenthétique des verbes épistémiques dans trois langues romanes
}

\author{
Gerda Haßler \\ Institut de romanistique \\ Université de Potsdam
}

\begin{abstract}
Dans cette contribution, nous étudierons le comportement syntaxique de verbes épistémiques français, espagnols et portugais qui permettent un emploi parenthétique dans toutes ces langues, mais avec des différences dans leur fréquence, dans leur emploi courant et dans leur position. Nous présenterons une analyse comparative, basée sur des corpus, du comportement syntaxique des verbes épistémiques dans les langues étudiées. Dans cette étude diachronique de l'usage parenthétique des verbes épistémiques, nous chercherons à savoir s'il y a eu une grammaticalisation dans laquelle les verbes auraient acquis un statut semblable à celui des adverbes. Dans un processus de grammaticalisation apparaissent de nouvelles unités dont la signification ne peut plus être déduite des éléments initiaux et qui ont éventuellement subi un processus de réduction ou de fusion. Ce n'est pas le cas des verbes épistémiques. II sera donc proposé de considérer le développement diachronique des verbes épistémiques comme un cas de pragmaticalisation, ce qui est confirmé par l'exemple du français, puisque I'usage parenthétique peut s'observer dans des textes anciens. Nous chercherons à démontrer l'hypothèse selon laquelle la fréquence de l'usage parenthétique, présent depuis longtemps dans le système de la langue, a été intensifiée par des conditions pragmatiques favorables.
\end{abstract}

Mots clés: parenthétique, verbes épistémiques, syntaxe, grammaticalisation, pragmaticalisation

This paper studies the syntactic behavior of French, Spanish and Portuguese epistemic verbs, which can be used parenthetically in all of these languages, but with differences in frequency and in their typical use and position. A comparative, corpus-based approach is pursued to examine the syntactic behavior of epistemic verbs in these languages. In this diachronic study of the parenthetical usage of epistemic verbs, the question is asked whether grammaticalization occurred in which the verbs took on a status similar to that of adverbs. In a process of grammaticalization, new units appear whose meaning can no longer be deduced from the initial elements and which may have undergone a process of reduction or fusion. This is not the case for epistemic verbs. It is therefore proposed to consider the diachronic development of epistemic verbs as a case of pragmaticalization, which is confirmed by the case of French, since parenthetical uses can be observed in Old French texts. We seek to demonstrate the hypothesis that the frequency of parenthetical usage, long present in the system of the language, has been intensified by favorable pragmatic conditions.

Keywords: parenthesis, epistemic verbs, syntax, grammaticalization, pragmaticalization

\section{L'usage parenthétique: tentative de définition}

Le concept de «parenthèse» a été traité dans la linguistique sous plusieurs dénominations et son contenu semble connaitre plusieurs interprétations possibles (voir Schneider, 2007: 3-I4). Dans le premier travail dédié entièrement aux parenthèses, Schwyzer (1939: 3I) les appelle des «phrases verbales parenthétiques» («parenthetische Verbalsätze») et 
Grevisse (1993: 273) utilisait le terme de «sous-phrase». Bolinger (1968) les considère comme des «phrases principales postposées» ("postposed main phrases») et Cornulier refuse la notion de "verbe parenthétique», tout en traitant ce type de phrase suivant la tradition française comme «incidente» (voir Cornulier, 1973).

Une parenthèse interrompt le déroulement de l'énoncé et elle est présentée comme secondaire par rapport au contenu de l'énoncé. Dans la langue parlée, ce caractère secondaire des parenthèses est marqué par l'intonation, notamment par une diminution de la fréquence, une absence de variation et une remontée de la fréquence à la fin de la parenthèse (Rouayrenc, 20IO: I23). Nous utilisons l'expression «usage parenthétique» au lieu de «parenthèse» pour deux raisons. Premièrement, les parenthèses peuvent être de nature diverse: elles sont le plus souvent des structures de type "pronom + syntagme verbal (plus ou moins long)», mais elles peuvent aussi consister en un syntagme prépositionnel restreignant la portée de l'énoncé (Rouayrenc, 20IO: I23). Nous étudions, en revanche, des verbes épistémiques accompagnés ou - en espagnol et en portugais - non accompagnés d'un pronom. Deuxièmement, il faut tenir compte du fait que les structures analysées peuvent apparaître aussi dans des phrases asyndétiques. Par construction asyndétique, nous entendons une phrase dans laquelle des mots de liaison sont absents ou supprimés. Tandis que les parenthèses peuvent s'insérer en divers points de l'énoncé, les asyndétiques entrent en relation de rection avec le reste de la phrase.

Nous entendons par usage parenthétique d'un verbe, son emploi en forme conjuguée qui peut être inséré librement dans une phrase et qui n'entre pas en relation syntaxique ouverte avec des éléments de celle-ci. Ce verbe peut alors perdre un argument requis par sa valence, mais cet argument peut être reconstruit à partir de la sémantique de la phrase dans laquelle l'expression parenthétique est insérée. Suivant Schneider (2007: 8), nous proposons six critères pour une expression parenthétique: la prosodie, l'interruption de la phrase, l'absence d'un lien perceptible entre la parenthèse et la phrase dans laquelle elle est insérée, malgré la présence d'une relation sémantique entre les deux, sa nature de phrase, sa fonction communicative.

Ainsi, syntaxiquement, l'expression parenthétique n'est ni une phrase principale ni une phrase subordonnée, mais elle est insérée ou adjointe comme pour un adverbe de phrase. Ainsi l'emploi de je crois dans la phrase [I] remplit-il la même fonction que l'adverbe probablement en [2], mis à part le fait qu'il souligne la perspective subjective du locuteur, qui est moins présente dans la modalisation par l'adverbe:

[I] Il est, je crois, le meilleur chanteur du pays.

[2] Il est probablement le meilleur chanteur du pays.

La position des expressions parenthétiques est libre et il n'y a pas de lien syntaxique avec la phrase dans laquelle elles sont insérées. Dans l'exemple [3], je crois ne gouverne pas les autres éléments de la phrase et cette séquence verbale peut être omise sans changer le sens propositionnel de la phrase. 
[3] C'est, je crois, techniquement possible.

(exemple extrait du web: la page, consultée le Is mars 20I2, n'est plus accessible)

Schneider (2007: 2) considère je crois dans [I] et [3] comme des phrases parenthétiques réduites et il les oppose à des phrases parenthétiques complètes dans lesquelles le verbe a tous les arguments exigés par sa valence:

[4] [...] on le notera je l'espère avec une grande ampleur sur le problème du ticket modérateur [...].

(Schneider, 2007: 2)

En effet, dans des phrases parenthétiques réduites, le verbe croire a une valence moindre que celle qu'il aurait dans un usage non parenthétique. Dans l'usage non parenthétique, il a, en dehors du sujet, un argument objet qui peut être réalisé par une phrase subordonnée introduite par que (exemple [5]), par un infinitif (exemple [6]) ou un groupe prépositionnel (exemple [7]):

[5] Par contre, je ne crois pas que ce soit ce gouvernement frileux de centre gauche qui fasse quelque chose à ce sujet.

(http://drogues.blog.lemonde.fr/2013/II/14/une-majorite-de-francais-en-faveurde-lautorisation-du-cannabis-sous-conditions)

[6] Franchement, je crois rêver en lisant ces lignes!

(http://emploi.blog.lemonde.fr/2013/II/II/dans-la-galere-quotidienne-dun-agentpole-emploi/\#xtor=RSS-3208)

[7] Je crois en une Roma compétitive.

(http://www.goal.com/fr/news/3o/italie/2013/og/20/4276712/ita-as-roma-totti-jecrois-en-une-roma-comp $\% \mathrm{C}_{3} \%$ Agtitive)

Tandis que croire, dans les exemples [5] à [7], apparaît comme verbe avec sa signification complète (Trésor de la langue française informatisé ${ }^{1}$ : "Attacher une valeur de vérité, ajouter foi à ce que dit une personne, tenir quelqu'un pour sincère, pour véridique; estimer vraies ses paroles»), dans l'usage en phrases parenthétiques réduites, il ne fait que relativiser la responsabilité du locuteur par rapport à la vérité de l'énoncé.

La réduction de la structure syntaxique par l'omission d'un argument et l'affaiblissement sémantique des phrases parenthétiques soulèvent le problème de savoir s'il s'agit de la grammaticalisation d'une phrase en un marqueur modal, ce que nous considérerons au cours de notre étude.

1. ATILF. Trésor de la langue française informatisé. En ligne à l'adresse suivante: http://atilf.atilf.fr/. 
Bien que les verbes épistémiques ne soient pas les seuls qui puissent suivre un usage parenthétique, ce sont les verbes sur lesquels les chercheurs se sont concentrés (voir Aijmer, 1997; Schneider, 2007; Posio, 2013). Les verbes épistémiques (par exemple croire, penser, trouver, avoir l'impression, savoir, voir, remarquer) ajoutent des éléments du savoir, de la croyance ou de la connaissance du locuteur à l'énoncé. Ce faisant, ils peuvent restreindre sa responsabilité quant au contenu ou bien souligner la subjectivité de l'énoncé. Nous parlons d'usage parenthétique des verbes épistémiques parce qu'ils peuvent être utilisés avec des fonctions bien différentes. Tandis qu'en tant que parenthèses ces verbes apparaissent comme adjoints, ils sont utilisés en position de tête dans d'autres cas (voir [5] à [7]).

Dans les dernières années, la recherche sur la contribution de la prosodie à la pragmatique de la communication a animé le débat sur les verbes épistémiques utilisés comme parenthétiques. Dehé (2007) et Barth-Weingarten et al. (2009) ont étudié les relations entre des parenthèses syntaxiques et prosodiques pour décrire les fonctions pragmatiques des expressions anglaises I think, I believe, I suppose. Quand ces "phrases commentatives» («comment clauses») sont séparées et accentuées, elles sont sémantiquement transparentes; c'est-à-dire qu'elles sont utilisées plutôt dans leur signification littérale. Leur intégration dans la phrase et leur désaccentuation, en revanche, s'accompagnent d'un affaiblissement sémantique. Ces phrases peuvent alors désigner l'incertitude du locuteur ou même jouer le rôle d'un "élément d'hésitation» («besitation phenomenon») et remplir une pause. Plusieurs auteurs (par exemple, Andersen, 1997; Féron, 2005; Féron Eusèbe, 1995; Blanche-Benveniste et Willems, 2007; Cappelli, 2007; Kärkkäinen, 2007; Barth-Weingarten et al., 2009) ont constaté que ces expressions courantes étaient en voie de grammaticalisation, comme les marqueurs discursifs.

Sur la base de ces études sur les verbes épistémiques utilisés parenthétiquement, on pourrait partir de l'hypothèse que ces verbes s'utilisent, dans la langue orale, surtout comme marqueurs modaux et qu'ils se trouvent dans un processus de grammaticalisation qui les rapproche fonctionnellement des adverbes modaux. Dans la grammaire générative, au contraire, on considère ces constructions incises comme des phrases matrices desquelles dépend une proposition subordonnée introduite par une conjonction non exprimée (Newmeyer, 20Io). Si l'on accepte cette position syntaxique, les constructions avec des verbes épistémiques devraient être décrites comme des énoncés performatifs exprimant, dans ce cas, l'acte de croire: Je crois $[q u e \rightarrow \varnothing]$ il est le meilleur chanteur du pays.

À partir d'études de corpus, nous avons analysé le comportement de verbes épistémiques espagnols, français et portugais pour vérifier ces deux hypothèses contraires et pour les examiner sous trois aspects. (I) L'usage parenthétique des verbes épistémiques est-il vraiment le résultat d'un changement linguistique ou constitue-t-il un fait de variation que l'on peut observer dès les premières étapes? (2) L'application du concept de grammaticalisation à ce processus est-elle justifiée ou serait-il préférable d'examiner ce processus en termes de polysémie et 
de pragmaticalisation? (3) Les constructions sans conjonction constituent-elles des cas de structures subordonnées ou des cas d'éventuelles constructions incises antéposées ${ }^{2}$ ?

Dans cette contribution, nous étudierons le comportement syntaxique de quelques verbes épistémiques français, espagnols et portugais, en nous concentrant sur le verbe qui exprime la croyance (je crois, creo, acho), qui permettent un emploi parenthétique, mais avec des différences dans leur fréquence, leur emploi courant, leur évolution historique et leur position. La comparaison de ces trois langues permet d'éviter de constater des phénomènes qui ne sont valables que pour une langue et d'expliquer l'essentiel de cet emploi des verbes épistémiques, tout en remarquant les spécificités de chaque langue. Cette analyse ne présuppose pas que ce soit l'origine commune des langues romanes qui permette l'usage parenthétique des verbes épistémiques. Elle attribue cette propriété à la sémantique de ces verbes et à son changement selon l'usage contextuel des verbes, ces deux éléments dépendant des activités cognitives.

L'analyse, qualitative, sera réalisée sur la base de corpus écrits. Nous commencerons par l'étude de la forme je crois en français, pour comparer ensuite les contraintes de l'usage parenthétique de ses équivalents espagnols et portugais dans une perspective diachronique. Les verbes désignant la croyance peuvent fonctionner comme des verbes épistémiques par excellence. Des verbes psychologiques factuels comme savoir, regretter, se réjouir présupposent la vérité de la proposition, tandis que des verbes épistémiques comme croire, penser, craindre ou espérer induisent la présupposition que la proposition introduite peut ne pas être pas vraie.

\section{L'usage parenthétique de je crois dans I'histoire du français : un phénomène de variation}

À partir des études sur l'usage des verbes parenthétiques, on pourrait formuler l'hypothèse que cet usage augmente dans le langage courant et que ces verbes sont en train d'être grammaticalisés et rapprochés, dans leur fonction, des adverbes modaux. Cette hypothèse présuppose un ancrage fonctionnel double de la notion de "grammaticalisation»: d'une part, elle se situe dans le paradigme fonctionnaliste de la linguistique qui pose que c'est dans l'usage même de la langue que s'initie le changement (voir Marchello-Nizia, 2006: I4-15), d'autre part elle vise le développement de formes qui remplissent une fonction grammaticale qu'on peut décrire comme étant de nature cognitive. L'expression de l'incertitude du savoir qu'on transmet par une énonciation modalisée peut être conçue comme une fonction grammaticale qui s'identifie sur une base cognitive. Sur cette base, il suffit de reconnaître qu'une phrase parenthétique a la même fonction (voir [I] et [2]) qu'un adverbe qui, de son côté, est le résultat d'un processus de grammaticalisation. Cela

2. Pour la discussion sur ces questions, voir Andersen (1997), Apothéloz (2003), Blanche-Benveniste et Willems (2007), Féron (2005), Récanati (1984) et Schneider (2007). 
aboutit à la naissance d'une nouvelle catégorie grammaticale d'adverbes en -ment, qui prend son origine dans le nom féminin latin mens.

On pourrait essayer d'expliquer l'usage d'un verbe conjugué avec son pronom sujet, mais sans lien explicite avec un autre argument, comme étant un effet de la variation en langue parlée. Julie Glikman décrit l'usage parenthétique ${ }^{3}$ de je crois en ancien français comme un phénomène de variation, celle-ci passant de «fréquente» à «fautive» (Glikman, 2009: 248-249; voir aussi Glikman, 2010):

Considérer la question de cette variation que/ $\varnothing$ dans le cadre d'une alternance langage parlé/langage écrit, telle qu'elle est définie dans Koch et Oesterreicher (200I), permet d'éclairer cette problématique et d'expliquer les différences de réalisation dans les textes ainsi que l'évolution du statut de cette alternance, qui est passée de «fréquente» à «fautive».

Pour le français, le fait que les verbes épistémiques soient suivis d'une phrase subordonnée introduite par que (que-P) avec une alternance en $\varnothing$-P, soulève plusieurs problèmes pour l'analyse syntaxique. Il faut pouvoir dire si les constructions sans conjonction constituent des cas de structures subordonnées ou, éventuellement, des cas de constructions incises antéposées (Glikman, 2009: I3I). Comme Glikman l'a montré, les verbes épistémiques en ancien français ont trois réalisations possibles. Dès l'ancien français, on peut les trouver aussi bien suivis d'une phrase subordonnée avec la conjonction que (que-P en [8]), qu'en construction incise (exemple [9]):

[8] Si est blecét, ne quit que anme i remaigne.

Il est blessé, je ne crois pas que l'âme y reste 4 .

(La chanson de Roland [entre 1075-1110], v. I848; Segre, 2003: 185)

[9] E si'n avrez, ço quid, de plus gentilz.

Et vous en aurez, je crois, de plus nobles.

(La cbanson de Roland [entre 1075-1110], v. 150; Segre, 2003: 100)

On les trouve aussi dans les constructions avec deux propositions subordonnées, comme on peut le voir dans l'exemple suivant:

[ı] «Ha! rois Pepins,» fait ele, «je croi mar vous vi né,

Quant on me veut murdrir delez vostre costé.»

Ab! roi Pépin, fait-elle, je crois que je vous vis né pour mon malheur,

puisqu'on veut me tuer à vos côtés.

(Adenet le Roi, Berte as grans piés, v. 425-426; Henry, 1982: 70)

En ce qui concerne la position des verbes épistémiques sans lien avec le reste de la phrase, Glikman (2009: 153) remarque que «les constructions apparaissant en

3. Sur la notion d'«emploi parenthétique», voir Andersen (1997) et Glikman (2009 et 2010).

4. Sauf mention contraire, toutes les traductions françaises des exemples sont de notre fait. 
fin de groupe prosodique [...] semblent bien être des constructions incises, analyse justifiée par le contexte et le fait que les constructions verbales dans cette position sont toujours à la première personne du présent». L'exemple de ço crei dans l'énoncé suivant montre ce comportement du verbe:

[II] Iert i sis niés, li quens Rollant, ço crei, E Oliver, li proz e li curteis.

Il y aura son neveu, le comte Roland, je crois, et Olivier, le preux et le courtois.

(La chanson de Roland [entre 1075-IIIO], v. 575-576; Segre, 2003: I2I)

5. Université de Leipzig. Corpus Wortschatz - Corpus français. En ligne à l'adresse suivante: http://wortschatz. uni-leipzig.de/ws_fra/index.php.

6. Université catholique de Louvain, Centre de traitement automatique du langage. Moteur de recherche GlossaNet. En ligne à l'adresse suivante: http://glossa.fltr.ucl.ac.be/. 
dans des textes conçus oralement, cette séquence verbale apparait aussi en tant que construction parenthétique. La construction parenthétique en français moderne est considérée comme fautive, mais elle continue d'exister à l'oral. Cela peut être démontré avec les données du Corpus Wortschatz dans lequel j’ai cherché la forme de la première personne du présent de l'indicatif du verbe épistémique croire: avec I67887 occurrences, que (que/ qu') est le voisin de droite le plus significatif de crois, suivi de pas avec 39655 occurrences qui sont normalement aussi suivies par que après la négation. Mais on peut néanmoins trouver des occurrences parenthétiques:

[I2] La situation actuelle du cinéma français n'est pas, je crois, sans portée pour notre réflexion commune d'aujourd'hui.

(http://www.culture.gouv.fr/culture/actualites/conferen/tasca-2002/imgeurope.htm)

[13] Il sera judicieux, je crois, d'apporter plusieurs changements dans mon onze de départ. (exemple extrait du web: la page, consultée le I2 mars 20I2, n'est plus accessible)

[I4] J'en ai déjà vaguement parlé, je crois, dans les pages précédentes, mais depuis mi-février, j’ai mené et remporté!

(exemple extrait du web: la page, consultée le I2 mars 20I2, n'est plus accessible)

Les exemples [I2] à [I4] relèvent évidemment de la langue parlée et ils confirment l'explication de cette structure comme phénomène du code oral. Les exemples [I2] et [I3] sont des jugements subjectifs du locuteur dont la validité est atténuée par je crois. Le locuteur choisit cette parenthèse pour formuler son jugement moins abruptement et il aurait pu utiliser d'autres moyens linguistiques pour le faire, par exemple des adverbes (probablement, certainement) ou une expression évidentielle (selon toute apparence). Cependant, la parenthèse je crois lui permet d'exprimer la restriction épistémique par une forme personnelle. Dans l'exemple [I4], je crois est utilisé pour renvoyer à la mémoire défaillante du locuteur et il souligne aussi un processus cognitif. Dans tous ces exemples, je crois n'exprime pas l'acte performatif d'une croyance, mais il ajoute une information modale sur l'attitude épistémique du locuteur.

\section{L'usage parenthétique de verbes épistémiques en espagnol et ses contraintes}

Les mêmes tendances peuvent être observées, avec un certain décalage dans le temps, pour l'espagnol et le portugais. Une étude sur les occurrences de creo dans le Corpus del Español (CDE) (Davies, 2002-), un corpus d'espagnol qui comprend Ioo millions de mots, a donné des résultats étonnants qui remettent en question les hypothèses de départ sur le développement et la grammaticalisation des verbes épistémiques.

Pour le XIII ${ }^{e}$ siècle, le corpus donne I59 occurrences de creo, dont seulement trois en usage parenthétique. Deux de ces occurrences parenthétiques sont extraites 
du Libro de Alexandre, daté de la première moitié du XIII ${ }^{e}$ siècle, qui raconte la vie d'Alexandre le Grand; par exemple:

[I5] Por que ouiessen agua; ca mester les era syno toda la hueste creo sele perdiera.

Pour qu'ils aient de l'eau, car ils en avaient besoin, sinon toute l'armée, je crois, serait perdue.

(CDE: Libro de Alexandre)

Dans l'exemple [I5], creo se met simplement avant le verbe et semble renforcer la subjectivité de l'énoncé et donner un centre déictique à son contenu. On ne peut pas exclure que les deux emplois parenthétiques, dans le Libro de Alexandre, soient inspirés d'un texte français, le Roman d'Alexandre, bien qu'ils ne soient pas traduits littéralement.

Dans l'exemple [16], il s'agit d'une traduction du français, la Gran conquista de Ultramar, un récit médiéval qui contient une chronique de la conquête de Jérusalem et qui fut rédigé entre I29I et I295. Dans cet exemple, l'omission de que après creo est peut-être en prise directe avec l'introduction de la phrase qui annonce une conséquence de la phrase antérieure. Le processus cognitif permettant de tirer cette conclusion est subjectivisé par l'interposition de la parenthèse creo:

[16] [...] ay buena gente que aman \& temen a dios mas que ningunas de las gentes que yo halle enlas tierras por do vine desde francia aca: \& porende creo han \& seran de ella reyes los dos destos tres hermanos [...].

[...] il y a de bonnes gens qui aiment et craignent Dieu, plus qu'aucune des personnes que j’ai pu rencontrer dans les terres par lesquelles je suis venu de la France jusqu'ici: et pour cette raison, je crois [que] deux des trois frères la possède et en seront les rois [...].

(CDE: Gran conquista de Ultramar)

Dans l'usage de creo, au XIII ${ }^{\mathrm{e}}$ siècle, la construction avec un complément d'objet domine nettement, soit avec un objet nominal ou pronominal (exemple [17]), soit avec une proposition subordonnée introduite par que (exemple [18]). L'usage parenthétique de creo, en revanche, est limité, à cette époque, à deux textes qui ont suivi des modèles français:

[17] Creolo.

Je le crois.

(CDE: Alfonso X, General estoria IV)

[18] Creo yo que todas las animalias que uiuen en las aguas ensaneldan. pero es de entender que cada una de su manera $\&$ segunt su natura.

Je crois que tous les animaux qui vivent dans l'eau respirent mais il faut comprendre que chacun le fait à sa manière et selon sa nature.

(CDE : Alfonso X, General estoria II) 
$\mathrm{Au} \mathrm{XIV}^{\mathrm{e}}$ siècle, l'usage parenthétique continue, mais d'une manière restreinte et chez des auteurs qui se sont inspirés de textes français. Les deux cas trouvés parmi les 406 occurrences de creo en témoignent nettement; ainsi : 
[22] «Cauallero, mal consejo ouistes en vos querer atreuer a lidiar comigo. Creo mejor fizierades en vos fincar en vuestra posada.»

"Monsieur, vous avez suivi un mauvais conseil en osant lutter contre moi. Je crois [que] vous feriez mieux $[$ de] rester dans votre maison.»

(CDE: Libro del Caballero Zifar, ca. I300)

est intéressant d'observer que l'usage de como creo n'est plus représenté au $\mathrm{XV}^{\mathrm{e}}$ siècle, ce qui pourrait être considéré comme un argument pour son rôle de préparateur à l'usage parenthétique de creo. Il y a, cependant, une autre locution introductive qui prend partiellement son rôle: según creo («selon ce que je crois»): 
[26] Por non creer según creo murio çesar cruel ment [...].

Pour ne pas croire, selon ce que je crois, [que] César est mort cruellement [...].

(CDE : Cancionero castellano de París)

Dans quelques types de textes, on trouve un usage renforcé de creo parenthétique. Cela se manifeste déjà dans l'Historia de la linda Melosina de Jean d'Arras, de I489, qui est une traduction espagnole du texte français dans laquelle des structures syntaxiques sont partiellement transposées dans la langue cible. Le traducteur a mis creo devant un verbe à l'indicatif (exemple [27]), ainsi que devant un verbe au subjonctif (exemple [28]), cette dernière option étant surtout choisie dans le cas de la négation du verbe parenthétique:

[27] Dize la ystoria que seyendo Remondin asy lleno de toda tristeza cayo en tiera como quien es trespasado aviendo tales dolores como creo suelen aver los que mas son çerca del punto dela muerte.

L’histoire dit que Remondin, étant plein de tristesse, tomba à terre comme celui qui est trépassé et qui avait des douleurs comme, je crois, ceux qui sont près de la mort en ont.

(CDE: Jean d'Arras, Historia de la linda Melosina)

[28] La qual no creo tornara en vuestra verguença mas en muy grand honor.

Celle-ci, je ne crois pas [qu] elle deviendra votre honte, mais un très grand bonneur.

(CDE: Jean d'Arras, Historia de la linda Melosina)

Un autre type de textes comprenant beaucoup d'occurrences de creo parenthétique correspond aux «traités utiles» de médecine, par exemple le Tratado útil e muy provechoso contra toda pestilencia e ayre corupto (1507, Traité utile et très bénéfique contre toute sorte de fétidité et air corrompu) de Licenciado Fores. Dans les lignes suivantes, l'auteur l'utilise trois fois, une fois accompagné d'une négation et du conditionnel, une fois accompagné d'une négation et du présent de l'indicatif et une troisième fois sans négation.

[29] La carne del carnero castrado \& merino fasta dos años no creo seria dañoso [...]. Uaca en tiempo caliente gruessa $\&$ no en mucha quantidad ni vieja no creo es dañosa señaladamente a buenos estomagos [...]. Dizese que son mejor assadas que no cozidas las dichas carnes por la humidad que se gana del cozimiento del agua esto creo deue ser segun regla general [...].

La viande du bélier châtré mérinos jusqu’à deux ans, je ne crois pas [qu] elle serait nuisible [...]. En temps chaud, du bouf gras et pas en grande quantité, je ne crois pas [que] ce soit nuisible, surtout aux bons estomacs [...]. On dit que ces viandes sont meilleures rôties que cuites, par l'bumidité qu'on gagne à faire bouillir de l'eau, cela, je crois, doit être une règle générale.

(CDE: Licenciado Fores, Tratado útil, 1507)

Ces emplois de creo ne contribuent pas à la prédication : on pourrait facilement les omettre sans changer le sens de la phrase. Ils modalisent les énoncés du point de 
vue du locuteur, mais ils ne mettent pas en question le contenu de la proposition. Ce faisant, ils se rapprochent de la fonction des marqueurs adverbiaux et discursifs. Un autre auteur qui utilise beaucoup ce creo parenthétique est Tomás de Mercado dans la Summa de tratos y contratos (I545), où nous avons I9 occurrences d'un tel emploi:

[30] De esta venta al fiado hemos de hablar más en particular y, creo, con más claridad, en el capítulo siguiente, do se entenderá mejor la verdad de éste.

De cette vente à crédit, il faut que nous parlions en détail et, je crois, avec plus de clarté, dans le chapitre suivant, où l'on comprendra mieux la vérité de cela.

(CDE: Tomás de Mercado, Summa de tratos y contratos, 1545)

Nous avons constaté que cet usage abondant de creo parenthétique est surtout caractéristique de textes qui portent un trait d'oralité, comme des livres de conseils pratiques, des lettres ou certains récits de voyages, sans être pour autant limité à ceux-ci. Il ne semble pas possible de déterminer les contraintes de l'usage parenthétique à partir de critères sémantiques et fonctionnels. On ne peut trouver qu'un seul critère distributionnel qui semble contraindre la chute du que dans presque tous les cas: la présence d'un autre que avant creo.

[3I] Queriendo pues imitar a éstos, que en afecto y obras fueron verdaderos padres, y mirando el estado presente de estos reinos y de todas las Indias, y que creo durará algunos siglos.

Voulant imiter ceux qui en disposition et en actes étaient de vrais pères et regardant l'état présent de ces royaumes et de toutes les Indes, [état] qui, je crois, durera quelques siècles.

(CDE: Tomás de Mercado, Summa de tratos y contratos, 1545)

Dans l'exemple [29], la conjonction que introduit une phrase relative dans laquelle creo marque une modalisation à partir du point de vue du locuteur. Dans cette position, le que après creo est systématiquement omis, certainement pour éviter une construction syntaxique trop lourde avec une subordination double. Cette contrainte semble fonctionner surtout à partir du XV siècle mais elle se manifeste aussi au XVI ${ }^{\text {e }}$ siècle, pour lequel nous avons pu relever 42 occurrences parenthétiques parmi les 306I occurrences de creo. À côté d'exemples qui suivent la contrainte distributionnelle décrite (exemple [3I]), il y a aussi des emplois libres de creo parenthétique (exemple [32]):

[32] Bien creo me faltará mucho para cumplir lo que esta materia demanda; pero harto será comenzar, y abrir el camino a los que fueren más diligentes y más avisados, y no menos servidores que yo, de vuestra Excelencia.

Je crois bien [qu] il me faudra beaucoup pour satisfaire à ce que cette matière exige, mais j'en ai assez de commencer et d'ouvrir le chemin à ceux qui sont plus forts et plus prudents, et pas moins serviteurs de Votre Excellence.

(CDE: Melchor de Santa Cruz, Floresta española, I546) 
Dans l'exemple [32], creo introduit des phrases graves par leur contenu, tout en les atténuant par sa fonction modalisante qui, dans ce cas, est soulignée par l'adverbe bien. Cet adverbe attribue un plus haut degré de probabilité à l'énoncé et il marque aussi la responsabilité du locuteur par rapport à son contenu.

La conjonction que est utilisée normalement par Cervantès, même dans le cas d'un que avant $c r e o$, que nous avons décrit comme contrainte de l'usage parenthétique:

[33] No sé qué diablo ha sido esto, ni por dónde se ha entrado este amor que le tengo, siendo yo tan muchacha y él tan muchacho, que en verdad que creo que somos de una edad mesma, y que yo no tengo cumplidos diez y seis años.

Et pourtant, je ne sais comment le diable s'en est mêlé, ni par où m'est entré cet amour que j’ai pour lui, étant, moi, si jeune fille, et lui, si jeune garçon: car, en vérité, je crois que nous sommes du même âge, et je n'ai pas encore mes seize ans accomplis.

(CDE: Cervantès, Don Quijote de la Mancha, I6o5; trad. fr.: L'ingénieux bidalgo Don Quichotte de la Manche, Bibliothèque électronique du Québec [À tous les vents; 294], p. 99I, en ligne à l'adresse suivante: http://beq.ebooksgratuits.com/cervantes/ Cervantes-I.pdf)

Ce que je viens de présenter pourrait mener à la conclusion que l'usage parenthétique apparait surtout dans des textes marqués par l'oralité et que l'usage littéraire continue à employer la conjonction. En effet, au XVII ${ }^{e}$ et au XVIII ${ }^{e}$ siècle, on trouve l'usage parenthétique systématiquement dans des lettres, des écrits non officiels, des récits de voyages. Ces textes sont réalisés à l'écrit, mais conçus comme s'ils étaient parlés. Ainsi, Francisco de Miranda utilise creo d'une manière parenthétique dans un texte politique et personnel en même temps:

[34] De esta suerte, creo, se cumple con la ley, se precaven los riesgos, se reparan en parte los males y perjuicios recibidos, se protege la inocencia, se castiga la culpa, y sobre todo, dará V. A. a los pueblos de Venezuela y al mundo entero un público testimonio de su imparcialidad y del carácter con que se halla revestida.

Ce bonbeur, je crois, s'accomplit avec la loi, les risques sont évités, le mal et les préjudices sont partiellement réparés, l'innocence est protégée, la culpabilité est punie, et surtout Votre Altesse donnera publiquement aux peuples $d u$ Venezuela et au monde entier une preuve de son impartialité et du caractère qu'elle présente.

(CDE: Francisco de Miranda, Escritos, 1783 )

Mais l'usage avec que se maintient de la même manière et dans la même position syntaxique chez les mêmes auteurs:

[35] Los edificios y casas de porte desente, no creo que llegasen á 3 dozenas en todo el Lugar; las demas son casucas y tendajos.

Les édifices et les maisons de portage décents, je ne crois pas qu'ils atteignent trois douzaines dans l'endroit, les autres sont des cabanes et des bouges.

(CDE: Francisco de Miranda, Diario desde New York, hacia Albany, Saratoga..., 1783) 
L'hypothèse d'un lien entre l'emploi parenthétique et la modalité orale se trouve, en revanche, complètement remise en question par l'étude de la partie orale du Corpus del Español, datée du XX $\mathrm{XX}^{\mathrm{e}}$ siècle, qui présente II 604 occurrences de creo. Hormis pour quelques exceptions qui représentent moins de $0,5 \%$ des occurrences de creo, la subordination avec que est utilisée. Ce constat vaut également pour l'usage très spontané et pour les variétés de l'espagnol parlées dans les pays hispanophones de l'Amérique latine. Dans l'exemple suivant d'une locutrice de Buenos Aires, la phrase commencée par yo creo que n'est pas terminée. La prononciation allongée de que lui permet de réfléchir et de recommencer par une phrase tout à fait nouvelle:

[36] Yo creo que... no sé si había ido con vos o sola, y [...].

Je crois que... je ne sais pas si elle était partie avec toi ou seule, et [...].

(CDE: oral, «Habla culta: Buenos Aires: M27 B»)

On peut même constater un usage massif du pronom sujet yo qui est normalement omis en espagnol, sauf si l'on veut le focaliser ou le mettre en contraste avec un autre sujet. Comment peut-on expliquer ce fait qui contredit toute explication du développement en termes de grammaticalisation et d'oralité de l'usage parenthétique? Selon moi, c'est la fonction pragmatique qui détermine ici l'usage d'une forme qui met le plus possible en valeur l'acte d'énonciation d'une opinion et sa subjectivité. Dans un énoncé sans conjonction, le locuteur expose un fait et le modalise secondairement; dans une phrase complexe avec subordination, il exprime primairement son opinion. La référence au locuteur comme porteur de l'énoncé et de son contenu peut être exprimée par plusieurs moyens linguistiques, comme, dans [35a], avec le pronom sujet, le verbe creo, la confirmation métadiscursive qu'il exprime son opinion personnelle et la superposition de cette structure entière comme phrase matrice à laquelle la proposition qui contient l'information principale est subordonnée:

[37a] Yo creo - es mi opinión personal - que Carmen Amaya no ha dejado herederos en el baile porque es irrepetible.

Je crois - c'est mon opinion personnelle - que Carmen Amaya n'a pas laissé d'béritiers dans la danse parce qu'elle est extraordinaire.

(CDE: Antonio Gades, $A B C$ )

Dans [37b], la séquence métadiscursive est éliminée, mais, par sa forme syntaxique, la phrase est toujours l'expression d'une opinion. Dans la communication réelle, cette structure ne correspond pas toujours à l'expression d'une subjectivité soulignée. Le locuteur peut introduire une phrase par yo creo que parce qu'il hésite dans sa formulation et parce qu'il a besoin de temps pour chercher ses mots.

[37b] Yo creo que Carmen Amaya no ha dejado herederos en el baile porque es irrepetible.

Dans [37c], l'introduction se réduit à creo que, le pronom sujet a disparu et cela donne une phrase sans mise en relief du sujet. Il s'agit, cependant, toujours d'une phrase complexe, dans laquelle l'information principale occupe la place de la proposition subordonnée: 
[37c] Creo que Carmen Amaya no ha dejado herederos en el baile porque es irrepetible.

Dans [37d], le verbe épistémique creo est utilisé en tant que parenthèse et ajoute une valeur modale au contenu de la phrase. Le locuteur subjectivise le degré de véracité, mais il le fait d'une manière plutôt subtile, sans se mettre au premier plan comme énonciateur:

[37d] Carmen Amaya, creo, no ha dejado herederos en el baile porque es irrepetible.

On trouve aussi des occurrences avec creo parenthétique et le pronom sujet postposé, c'est-à-dire en position rhématique, qui soulignent la subjectivité de l'énoncé:

[38] Este año va a pasar lo mismo, creo yo.

Cette année la même chose va arriver, je crois.

(CDE : oral, «Habla culta: Bogotá: $\mathrm{M}_{34}$ )

Le développement qui commence par la variation entre la subordination avec que et l'usage parenthétique et qui finit avec une préférence nette pour la subordination, est confirmé par les données pour la forme pienso, pour laquelle nous avons relevé 7386 occurrences dans le corpus, à peu près un tiers des 23600 occurrences trouvées pour creo. L'usage parenthétique de pienso est très répandu depuis le XVI ${ }^{e}$ siècle et il connait toutes les positions:

[39] Esto también nos llegó, pienso, caminando con pasos rápidos y sin dejar de fumar. Celui-là nous est aussi arrivé, je pense, marchant à pas rapides et sans cesser de fumer.

(CDE: Augusto Casola, La catedral sumergida)

[40] Eso es lo que me va mejor, pienso.

C'est ce qui me va mieux, je pense.

(CDE : Carlos Semprún Maura, interview $[A B C]$ )

La signification de pensar implique un processus cognitif dirigé vers un but, ce qui est complètement absent de la signification de creer et c'est ce qui s'oppose probablement à sa grammaticalisation partielle comme particule modale. Dans certains contextes, pienso exprime même d'une manière prépondérante l'intention $\mathrm{du}$ locuteur, c'est-à-dire une modalité volitive qui n'est pas compatible avec la modalité épistémique. Cet usage de pienso apparaît souvent avec la négation (no pienso bacer daño alguno, «je ne pense pas causer un préjudice»; tampoco pienso ir a Puerto, «moi non plus je ne pense pas aller à Puerto»), mais aussi comme expression de la volonté de faire quelque chose (pienso traerlo al escritorio, «je pense le porter au bureau»; pienso ir a Ibiza, «je pense aller à Ibiza»; pienso escribir cien novelas de pura invención, «je pense écrire cent romans de pure invention») ou avec le but de la pensée comme complément d'objet prépositionnel de pienso (pienso en el fin, «je pense à la fin»; pienso en el futuro, «je pense à l'avenir»). Dans tous ces cas, l'usage 
de creo au lieu de pienso serait impossible ou comporterait une modalité d'insécurité très forte. C'est pourquoi creo est l'élément prédestiné à une grammaticalisation comme particule modale qui, comme on l'a vu, n'est cependant pas très avancée.

Il serait, peut-être, plus adéquat de dénommer le processus que ces verbes ont subi par le terme de pragmaticalisation. Nous considérons la pragmaticalisation comme un processus dans lequel un élément est sémantiquement affaibli et acquiert une fonction discursive qui ne concerne pas la véracité de l'énoncé?

\section{L'emploi parenthétique tardif de creio et acho}

Le Corpus do Português (CDP) (Davies et Ferreira, 2006-), un corpus de portugais qui compte 45 millions de mots, confirme ce que nous avons constaté pour l'espagnol, mais avec un autre décalage dans le temps et une attribution de la même fonction à deux verbes d'origine étymologique différente (crer, «croire»; achar, «trouver»). $\mathrm{La}$ forme verbale creio n'apparaît qu'au $\mathrm{XV}^{\mathrm{e}}$ siècle comme matrice d'une proposition subordonnée introduite par la conjonction que:

[4I] E porque no joguo avya preço, o qual creio que era perdizes, dise-lhe meu criado que se calase [...].

Et parce que, dans le jeu, il y avait un prix, je crois que c'étaient des perdrix, j’ai dit à mon serviteur qu'il se taise [...].

(CDP : Cronica de Portugal, I419)

$\mathrm{Au} \mathrm{XVI}{ }^{e}$ et au XVII ${ }^{e}$ siècle apparait une structure comparative avec como eu creio, parallèle à celle que j'ai mentionnée pour l'espagnol (como creo):

[42] E se ele vos requer e pede já isto mesmo, como eu creio, não seja parte desmerecermolo nós para vos deixardes de o ouvir a ele.

Et s'il vous sollicite et vous demande vraiment cela, comme je crois, il nous revient de ne pas démériter pour que vous continuiez à l'entendre.

(CDP : João de Lucena, Historia da vida do Padre S. Francisco Xavier, 1600)

Ce n'est qu'à partir du XIX ${ }^{\mathrm{e}}$ siècle qu'on peut relever des exemples d'usage parenthétique des formes verbales creio et acho. Ils apparaissent dans des textes littéraires qui utilisent l'oralité simulée dans les dialogues des personnages et ils sont suivis du pronom personnel de la première personne:

7. "If we differentiate between semantics and pragmatics in trutb-conditional terms, as is common, we can stipulate that elements which cannot be analysed in terms of truth are pragmatic or pragmaticalized» (Aijmer, 1997:3; «Si nous distinguons la sémantique de la pragmatique en termes de conditions de vérité, comme on le fait d'habitude, nous pouvons stipuler que des éléments qui ne peuvent pas être analysés en termes de vérité sont pragmatiques ou pragmaticalisés»). Une autre solution a été proposée par Ilse Wischer qui parle d'une grammaticalisation discursive. Sur l'étude des parenthétiques en termes de grammaticalisation, voir aussi Fischer (2007), Brinton (1996) ainsi que Thompson et Mulac (I991). 
[43] E, depois de pensar um momento, acrescentou:

- De Espanha, acho eu... Era, era de Espanha...

Et après avoir pensé un moment, il ajouta:

- D’Espagne je crois... Il était, il était d'Espagne.

(CDP : Júlio Dinis, Uma família inglesa)

[44] "Il ne sera plus beau garçon» disse o parlamentário francês que veio, depois da acção, tratar, creio eu, de troca de prisioneiros ou de coisa semelhante.

"Il ne sera plus beau garçon» dit le parlementaire français qui vint, après l'action, je crois, changer des prisonniers ou quelque chose de semblable.

(CDP : Almeida Garrett, Viagens na minha terra, 1846)

Dans la langue contemporaine, l'emploi de acho sans que est extrêmement rare. Il relève du langage oral et le verbe est suivi par le pronom personnel $e u$ («moi») postposé:

[45] É um rico, que ele não precisa daquilo; é já para fazer mal a nós. Porque tem havido reclamações. Acho, acho eu, que ele tem compadres que tapem em Portimão. Acho $e u$, porque oiço dizer.

C'est un riche parce qu'il n'a pas besoin de ça, il veut nous faire mal. Parce qu'il avait des plaintes. Je, je crois, qu'il a des compères qui agissent à Portimão. Je crois, parce que j’ai oü dire.

(CDP : oral, «Cordial: ALVo2»)

L'emploi parenthétique de creio est plus fréquent, mais restreint à l'usage oral ou à l'oralité simulée.

[46] No futuro, creio, isso será possível.

Dans le futur, je crois, cela sera possible.

(CDP : oral, Ciro Gomes)

[47] O facto de se tratar de um debate concentrado em três dias terá, creio, virtualidades próprias, pois é a única forma de reunir as individualidades que vão participar num diálogo que será profícuo. E além de novas ideias para o desenvolvimento, creio que o EuroNorte vai reforçar a consciência colectiva da importância desta grande região económica.

Le fait qu'il s'agit d'un débat concentré sur trois jours aura, je crois, ses propres vertus, car c'est le seul moyen de réunir des personnes qui vont participer à un dialogue qui sera fructueux. Et en dehors d'idées nouvelles pour le développement, je crois que l'EuroNorte va renforcer la conscience collective de l'importance de cette grande région économique.

(CDP : oral, Ludgero Marques)

L'exemple [47] présente un usage parenthétique entre le verbe et le complément d'objet, qui modalise la réalité de ces «virtualidades próprias». Dans la phrase suivante, 
la modalisation se réfère à toute la proposition et elle est exprimée par l'emploi de creio avec subordination conjonctive.

Creio est utilisé en tant que parenthèse avec les variantes eu creio et creio eu:

[48] E, de facto, essa questão dos alunos, hoje em dia, darem pouca importância à participação tem um pouco a ver com a descrença, eu creio, com o facto de, hoje em dia, por exemplo, quando se fala em políticos há logo uma carga negativa.

En fait, cette question des élèves, qui de nos jours attribuent peu d'importance à la participation, a un peu à voir avec la méfiance, je crois, avec le fait que, aujourd'bui, par exemple, quand on parle d'hommes politiques il y a tout de suite une connotation négative.

(CDP : oral, Ana Mesquita)

[49] Passou-se, creio eu, no tempo em que os animais falavam.

Cela se passait, je crois, à l'époque où les animaux parlaient.

(CDP : Alexandre Cabral, Margem norte, 1979)

En portugais, la construction avec que après creio est dominante. Comme nous l'avons vu pour l'espagnol, l'accumulation de plusieurs formes que, avec des fonctions différentes, était une des raisons d'omettre le que après creo. En portugais, ce n'est pas le cas, la subordination conjonctive est choisie même s'il y a un autre que très proche dans le contexte:

[5o] Creio que é algo que a Conferência acabará por consagrar.

Je crois que c'est quelque chose que la Conférence approuvera finalement.

(CDP : oral, Francisco Seixas da Costa)

Cette subordination avec que a l'avantage de permettre le marquage de l'acte de l'expression de l'opinion ainsi que la thématisation de cette partie de l'énoncé. Nous entendons par thème la partie de l'énoncé qui correspond au point de départ de la communication. Ce sujet dont on parle peut être une information connue grâce au contexte ou à la situation dans laquelle il est présenté explicitement comme tel par le locuteur, par exemple par une dislocation à gauche. Dans la phrase en [5I], la partie mise en italique est le thème de l'énoncé, dans lequel il y a trois références au locuteur: la forme verbale creio, le pronom accentué mim et sa reprise par le pronom clitique me. La subordination par que accentue ce poids du thème:

[51] Creio que, a mim, o que me comove no Natal é ainda a minha infância, apesar de eu quase não ter tido infância...

Je crois que moi, ce qui m'émeut à Noël, c'est toujours mon enfance, bien que je n'aie presque pas eu d'enfance...

(CDP: Urbano Tavares Rodrigues, Os insubmissos, 1976) 
Dans l'exemple [52], la formule o que eu acho é que sert à introduire le thème de la phrase, qui est caractérisé comme l'opinion du locuteur:

[52] O que eu acho é que nos meios das legislaturas, nomeadamente quando há eleições, é preciso tirar conclusões.

Ce que je crois est que, au milieu de la durée du mandat, notamment quand il y a des élections, il est nécessaire de tirer des conclusions.

(CDP : oral, Jorge Coelho)

La subordination avec que permet de raccourcir un énoncé à la particule d'affirmation ou de négation, ce qui ne semble pas possible sans conjonction. Le corpus donne 78 occurrences pour acho que sim («je trouve que oui») et 66 pour creio que sim («je crois que oui»), 179 occurrences pour creio que não et 225 pour acho que não, tandis que la structure courante en anglais I think yes («je crois [que] oui») n'est pas habituelle en portugais. Nous n'avons trouvé aucune occurrence pour creio sim ni pour creio não, et seulement six pour acho não et une pour acho sim. Ce sont toutes des occurrences de la langue parlée du Brésil. Cette dernière (acho sim) apparaît dans une interview avec un Brésilien qui vit aux États-Unis et qui peut être influencé par l'anglais. Si la tendance générale privilégie les énoncés courts, on voit que parmi les deux formes avec ou sans subordination, la forme avec la subordination en que est préférée.

[53] Então percorreu toda a casa?

- Creio que sim.

Alors, a-t-il parcouru toute la maison?

- Je crois que oui.

(CDP : Marcos Rey, Os crimes do Olbo-de-Boi, 1995)

[54] Serão necessários mais professores de Educação Física?

- Acho que não.

Est-ce qu'on aura besoin de plus de professeurs d'éducation physique?

- Je ne crois pas.

(CDP : oral, conseil d'administration, professeur Pedro)

Le poids argumentatif augmente grâce à la subordination avec la conjonction qui peut être répétée après la particule affirmative:

[55] Acho que sim, que poderás escrever um dia.

Je crois que oui, que tu pourras écrire un jour.

(CDP : Rita Ferro, Por tudo e por nada: crónicas, 1994)

En outre, la conjonction que peut être refonctionnalisée comme un élément d'hésitation (Koch et Oesterreicher, 20II : 54). Dans l'exemple [56], que est prononcé d'une manière dilatée et suivi d'une pause; dans [57], il est séparé de acho par une phrase métacommunicative: 
[56] X: Já vi cá golas altas. Creio que...

B: Mas olha que em direito em lisboa com alguns professores eu sei que era assim!

$X$ : J'y ai déjà vu des cols roulés. Je crois que...

B: Mais écoute, en droit à Lisbonne, c'était comme ça avec quelques professeurs.

(CDP : oral, «Corpus-Ref-Port-Contemp : 763»)

[57] Eu acho... - digo-o fatigadamente - que a matéria plástica com que um escritor lida é a língua.

Je trouve... - je le dis d'une manière surmenée - que la matière plastique qu'un écrivain traite est la langue.

(CDP : oral, Mário de Carvalho)

Le fait que l'usage de creio et de acho avec la conjonction que prédomine largement, y compris dans la langue parlée, peut s'expliquer, comme nous l'avons déjà vu pour l'espagnol, par un phénomène pragmatique. La construction avec que donne plus de poids à l'acte de langage qui consiste en l'expression de son opinion, tandis que la construction parenthétique ne peut que modaliser une proposition. Pour la modalisation, on dispose d'autres moyens linguistiques avec les adverbes. Évidemment, les verbes parenthétiques n'ont pas subi de processus de grammaticalisation qui les en rapprocherait.

\section{Perspectives et conclusion}

Sans pouvoir approfondir l'analyse sur d'autres verbes épistémiques, je voudrais terminer par un bref aperçu sur les verbes qui signifient «craindre». Ces verbes peuvent, dans certains contextes, acquérir une signification épistémique, en plus de leur signification évaluative. Ainsi par exemple, dans l'énonciation en [58a], on pourrait remplacer je le crains par je crois en gardant la signification modalisante, tout en perdant l'évaluation négative de la situation. Dans le corpus français, on peut identifier quelques phrases parenthétiques qui incluent l'objet pronominal le à je crains:

[58a] Et je n'étais pas le seul, je le crains, dans cette situation.

(Frantext: Jean d'Ormesson, Tous les hommes sont fous [1986], Paris, Librairie générale française, 1989, p. 267)

[58b] Et je n'étais pas le seul, je crois, dans cette situation.

Cette formulation peut être constatée, dans Frantext, dans des textes depuis le XVIII siècle:

[59] J'espère que le général ratifiera la caution de ses frères: mais il me hära, je le crains. (Frantext: abbé Prévost, Nouvelles lettres angloises ou Histoire du chevalier Grandisson [1755], Amsterdam - Paris, Hôtel Serpente, 1784, p. 222) 
[6o] Je suis criminel, je le crains, et je m'avançois témérairement au-devant de son glaive! (Frantext : Louis-Sébastien Mercier, Jezennemours: roman dramatique, Amsterdam, 1776, p. 219)

Pour l'espagnol, il y a plusieurs exemples de l'usage de temo sans conjonction subordinative depuis le $\mathrm{XV}^{\mathrm{e}}$ siècle :

[6I] Temo abrán de padecer nuestras almas sin remedio [...].

Je crains que nos âmes auront à souffrir sans remède [...].

(CDE: Juan Boscán, Obra completa, I490)

[62] [...] vuelta a la materna, y antigua patria, cuyo bien poseo, que ya gozo la gloria, temo, estoy helado y ardo, busco la paz [...].

[...] retour à la patrie maternelle et antique, dont je possède le bien et dont je jouis déjà de la gloire, je crains, je suis froid et fougueux, je cherche la paix [...].

(CDE: Vicente Espinel, Poesía, i587)

[63] A lo que respondió Sancho: «Yo soy, señor, tan desgraciado, que temo no ha de llegar el día en que en tal ejercicio me vea».

Sancho répondit à cela: «Je suis, Monsieur, tellement malbeureux que, je crains, le jour va arriver où vous me verrez dans un tel exercice».

(CDE : Cervantès, Don Quijote de la Mancha, I605)

Comme on le voit à partir des exemples [6r] à [63], l'usage de temo n'entraine pas le subjonctif dans la phrase suivante, ce qui peut être considéré comme une preuve de son indépendance. Cet usage parenthétique, parfois avec la forme pronominalisée (exemple [65]), se trouve encore aujourd'hui à côté de la subordination conjonctive :

[64] Si no la obtuvieran, temo, al menos no deberíamos descartarlo, que podrían tratar de liquidar a Roberto.

S'ils ne l'obtenaient pas, je crains, nous ne devrions au moins pas exclure qu'ils pourraient essayer d'achever Roberto.

(CDE : Ricardo Ludovico Gulminelli, Fecundación fraudulenta)

[65] Tal vez por ello es por lo que, me temo, falle nuestra capacidad de competir, no tanto en el mercado de las mercancías, sino en el mercado mucho más nutritivo de las ideas.

C'est peut-être pour cela que, je crains, notre capacité de concurrencer ne fonctionne pas, pas tant sur le marché des marchandises que sur le marché plus nutritif des idées.

(CDE: Blanca Berasategui, interview $[A B C]$ )

La comparaison entre le français, l'espagnol et le portugais a montré un parallélisme qui ne s'explique pas par la parenté entre ces trois langues romanes, mais par le 
potentiel sémantique des verbes épistémiques qui permet l'usage de ces verbes en différentes positions syntaxiques et avec différentes significations. Le fait que la valeur sémantique de ces verbes est presque identique s'explique certainement par leur base cognitive commune.

Cependant, l'analyse contrastive a montré aussi des différences entre les trajectoires des trois langues. Nous avons vu que, dans l'histoire des trois langues romanes, on peut parler d'une variation de l'usage recteur et de l'usage parenthétique des verbes épistémiques qui commence à des époques différentes. Tandis que les parenthèses sont courantes en ancien français, elles n'atteignent leur apogée qu'au XVI ${ }^{e}$ siècle en espagnol et en portugais. L'usage des parenthèses dans la langue orale joue un certain rôle dans les trois langues et elles sont utilisées pour créer de l'oralité simulée, par exemple dans des dialogues d'œuvres littéraires. Mais ce trait oral ne mène pas obligatoirement à les considérer comme fautives. Pour l'espagnol, Juan de Valdés, dans son Diálogo de la lengua (écrit vers I535 mais publié en 1737), avait même conseillé d'omettre le que après creo quand il est superflu:

[66] De refrán no se me ofrece ninguno que tenga este que demasiado, y creo lo causa la brevidad con que stán escritos; pero, si miráis en lo que leéis, hallaréis ser verdad lo que os digo en partes semejantes que ésta: «creo que será bien hazer esto» adonde aquel que stá superfluo, porque diría mejor: «creo será bien hazer esto».

De proverbe, il n'y en a aucun qui me vienne à l'esprit et qui ait ce que de trop, et je crois, cela se doit à la brièveté avec laquelle ils sont écrits; mais, si vous faites attention à ce que vous lisez, vous remarquerez que c'est vrai ce que je vous dis, dans des phrases semblables à celle-ci: «je crois qu'il sera bien de faire cela» où ce que est superflu, parce qu'elle serait mieux formulée: «je crois il sera bien de faire cela».

(CDE: Juan de Valdés, Diálogo de la lengua)

Comme l'exemple [19] le suggère, il est fort probable que l'usage des verbes épistémiques soit le résultat d'une abréviation d'une formule parenthétique comparative qui établit un lien explicite avec le locuteur. Ce procédé peut être identifié dans les trois langues (voir le tableau ci-dessous).

Dans l'usage parenthétique des verbes qui désignent la croyance, la signification modalisante est aujourd'hui dominante au préjudice du trait de l'«action d'exprimer ou d'avoir une opinion». Ainsi, le locuteur est relégué au second plan en faveur de l'expression d'une modalisation de la prédication. On pourrait supposer, avec Aijmer (2002), que les formes parenthétiques sont sur la voie d'une grammaticalisation vers des adverbes modaux, s'il n'y avait pas le facteur de leur faible quantité.

Ce qui mène à la décroissance de l'usage parenthétique des verbes épistémiques qui désignent la croyance en espagnol et en portugais, est surtout leur faible puissance dans l'expression d'une opinion personnelle. Celle-ci est plus grande dans les constructions avec la conjonction subordinative qui permet de modifier la structure informationnelle et d'accentuer l'énonciateur. De plus, dans ces langues, 
des verbes épistémiques exprimant aussi une évaluation de la proposition prennent en partie le rôle des verbes qui désignent la croyance dans les parenthèses. Il reste à mener une étude pour savoir si cette substitution partielle contribue à un changement de leur signification.

L'étude sur les corpus des trois langues a permis de reconnaitre qu'il est difficile de parler de grammaticalisation, même s'il y a des cas où je crois, creo et creio/acho remplissent la même fonction qu'un adverbe. Il y a un contre-courant très fort à la grammaticalisation qui consiste en l'usage des verbes épistémiques en tant que verbes performatifs qui expriment l'opinion du locuteur.

\begin{tabular}{|c|c|}
\hline [comme je crois] & $\rightarrow$ [je crois] \\
\hline $\begin{array}{l}\text { «Car on dit (vu sa corporance) / Que ce eût été } \\
\text { un maître bœuf. / Ci-gît Guion, Pape jadis, et } \\
\text { Roi: / Roi de surnom, Pape par fantaisie, / } \\
\text { Non marié, de peur (comme je crois) / D’être } \\
\text { cocu ou d'avoir jalousie.» (Frantext: Clément } \\
\text { Marot, L'adolescence clémentine [1538], Paris, } \\
\text { Librairie générale française, 2005, p. 230-23I) }\end{array}$ & $\begin{array}{l}\text { "Je ne sçay si je vous ay escrit que nous } \\
\text { avons icy un homme qui vient de Provence } \\
\text { et, je crois, d'Italie, qui après avoir avalé } \\
\text { Io ou I2 pots d'eau, fait une fontaine de sa } \\
\text { bouche [...].» (Frantext: Marin Mersenne, } \\
\text { Correspondance [1639], Paris, Éditions du } \\
\text { CNRS, t. VIII, I638-1639, I963, p. } 724 \text { ) }\end{array}$ \\
\hline [como creo] & $\rightarrow$ [creo $]$ \\
\hline $\begin{array}{l}\text { "Algunos cuemo creo. seen en angostura.» } \\
\text { (CDE: Libro de Alexandre, XIII siècle) }\end{array}$ & $\begin{array}{l}\text { «[... \& porende creo han \& seran de ella reyes } \\
\text { los dos destos tres hermanos [...].» (CDE: } \\
\text { Gran conquista de Ultramar, fin XIII" siècle) }\end{array}$ \\
\hline [como eu creio] & $\rightarrow$ [creio eu $]$ \\
\hline $\begin{array}{l}\text { «E se ele vos requer e pede já isto mesmo, } \\
\text { como eu creio, não seja parte desmerecermo- } \\
\text { lo nós para vos deixardes de o ouvir a ele.» } \\
\text { (CDP: João de Lucena, Historia da vida do } \\
\text { Padre S. Francisco Xavier, I6oo) }\end{array}$ & $\begin{array}{l}\text { "Il ne sera plus beau garçon" disse o parla- } \\
\text { mentário francês que veio, depois da acção, } \\
\text { tratar, creio eu, de troca de prisioneiros ou de } \\
\text { coisa semelhante.» (CDP: Almeida Garrett, } \\
\text { Viagens na minba terra, } 1846 \text { ) }\end{array}$ \\
\hline
\end{tabular}

\section{Références}

\section{Études}

Aijmer, K. 1997. I Think - An English Modal Particle. In T. Swan et O.J. Westvik (éd.), Modality in Germanic Languages: Historical and Comparative Perspectives. Trends in linguistics - Studies and monographs 99. Berlin - New York: Mouton de Gruyter : 1-48.

Aujmer, K. 2002. English Discourse Particles. Evidence from a Corpus. Studies in corpus linguistics Io. Amsterdam: J. Benjamins.

Andersen, H.L. 1997. Propositions parenthétiques et subordination en français parlé. Thèse de doctorat non publiée. Université de Copenhague.

Аротне́LOz, D. 2003. La rection dite «faible»: grammaticalisation ou différentiel de grammaticité? Verbum 25 (3):24I-262. 
Barth-Weingarten, D., Dehé, N. et Wichmann, A. (éd.). 2009. Where Prosody Meets Pragmatics. Studies in pragmatics 8. Bingley: Emerald.

Blanche-Benveniste, C. et Willems, D. 2007. Un nouveau regard sur les verbes «faibles». Bulletin de la Société de linguistique de Paris I02 (I): 217-254.

Bolinger, D. 1968. Postposed Main Phrases: An English Rule for the Romance Subjunctive. Canadian Journal of Linguistics $\mathrm{I4}:$ 3-30.

Brinton, L.J. 1996. Pragmatic Markers in English: Grammaticalization and Discourse Functions. Topics in English linguistics 19. Berlin - New York: Mouton de Gruyter.

CAPPELli, G. 2007. «I Reckon I Know How Leonardo da Vinci Must Have Felt... ». Epistemicity, Evidentiality and English Verbs of Cognitive Attitude. Pari: Pari Publishing.

Cornulier, B. (de) 1973. Considérations illustrées de quelques arbres et enrichies de plus de mille exemples sur les incises en français contemporain. Thèse de doctorat. Université de Provence.

DehÉ, N. 2007. The Relation between Syntactic and Prosodic Parenthesis. In N. Dehé et Y. Kavalova (éd.), Parentheticals. Linguistik aktuell ıo6. Amsterdam: J. Benjamins: 26I-284.

FÉRON, C. 2005. Modalisation et verbes d'opinion: quelques remarques sur croire, cuidier et penser en usage direct dans La Queste del Saint Graal. L'information grammaticale I04: I5-2I.

FÉRON EusèBe, C. 1995. Sémantique et syntaxe de quelques verbes épistémiques du moyen français: vers un traitement lexicographique. Lille: Atelier national de reproduction des thèses.

Fischer, O. 2007. The Development of English Parentheticals : A Case of Grammaticalization? In U. Smit et al. (éd.), Tracing English Through Time: Explorations in Language Variation - In Honour of Herbert Schendl on the Occasion of His 65th Birthday. Austrian studies in English 95. Vienne: Braumüller: 99-II4.

Glikman, J. 2009. Parataxe et subordination en ancien français. Système syntaxique, variantes et variation. Thèse de doctorat. Université Paris Ouest Nanterre La Défense - Université de Postdam. En ligne à l'adresse suivante: http://opus.kobv.de/ubp/volltexte/2011/5678/.

Glikman, J. 2oio. Peut-on établir des critères formels de reconnaissance de la parataxe: l'apport de l'ancien français. In M.-J. Reichler-BÉGuelin, M. Avanzi et G. Corminboeuf (éd.), La parataxe. Berne - Berlin - Bruxelles: P. Lang. T. I: Entre dépendance et intégration: $355^{-371 .}$

Grevisse, M. 1993. Le bon usage: grammaire française. Paris - Louvain-la-Neuve : De BoeckDuculot [ $\left[3^{\mathrm{e}}\right.$ éd., refondue par A. Goosse].

KärkкÄInen, E. 2007. The Role of I Guess in Conversational Stancetaking. In R. ENGLEBRETSON (éd.), Stancetaking in Discourse. Subjectivity, Evaluation, Interaction. Amsterdam - Philadelphie: J. Benjamins: 183-219.

Koch, P. et Oesterreicher, W. 200I. Gesprochene Sprache und geschriebene Sprache/Langage parlé et langage écrit. In C. Schmitt, M. Metzeltin et G. Holtus (éd.), Lexikon der romanistischen Linguistik. Tübingen: Niemeyer. T. I: 584-627.

Koch, P. et Oesterreicher, W. 2oir. Gesprochene Sprache in der Romania: Französisch, Italienisch, Spanisch. Berlin - New York: De Gruyter.

Marchello-Nizia, C. 2006. Grammaticalisation et changement linguistique. Bruxelles: De Boeck-Duculot. 
NewmeYer, F.J. 20Io. What Conversational English Tells Us about the Nature of Grammar: A Critique of Thompson's Analysis of Object Complements. In K. Boye et E. EngBerGPedersen (éd.), Language Usage and Language Structure. Berlin - New York: Mouton de Gruyter: 3-44.

Posio, P. 20I3. Subject Expression in Grammaticalizing Constructions: The Case of Creo and Acho «I think» in Spanish and Portuguese. Journal of Pragmatics 63: 5-18.

RÉCAnati, F. 1984. Remarques sur les verbes parenthétiques. In P. Attal et C. Muller (éd.), De la syntaxe à la pragmatique: actes du colloque de Rennes, université de HauteBretagne. Amsterdam - Philadelphie: J. Benjamins: 319-352.

RouAYrenc, C. 20IO. Le français oral. 2. L'organisation et la réalisation de l'énoncé oral. Paris: Belin.

SCHNEIDER, S. 2007. Reduced Parenthetical Clauses as Mitigators: A Corpus Study of Spoken French, Italian and Spanish. Amsterdam - Philadelphie: J. Benjamins.

SchWyzer, E. 1939. Die Parenthese im engern und weitern Sinne. Berlin: Verlag der Akademie der Wissenschaften.

Thompson, S.A. et Mulac, A. 199I. A Quantitative Perspective on the Grammaticalization of Epistemic Parentheticals in English. In E.C. Traugott et B. Heine (éd.), Approaches to Grammaticalization. Amsterdam - Philadelphie: J. Benjamins. Vol. 2: Focus on Types of Grammatical Markers: 313-329.

Wischer, I. 2000. Grammaticalization versus Lexicalization: «Methinks» There Is some Confusion. In O. Fischer, A. Rosenbach et D. Stein (éd.), Pathways of Change. Grammaticalization in English. Amsterdam - Philadelphie: J. Benjamins: 355-370.

\section{Textes et corpus cités}

Adenet le Roi. Berte as grans piés. Henry, A. (éd.) 1982. Textes littéraires français 305. Genève: Droz.

ATILF. Base textuelle Frantext. En ligne à l'adresse suivante: http://www.frantext.fr/.

Davies, M. 2002-. Corpus del Español. En ligne à l'adresse suivante: http://www. corpusdelespanol.org.

Davies, M. et Ferreira, M. 2006-. Corpus do Português. En ligne à l'adresse suivante: http://www.corpusdoportugues.org.

La chanson de Roland. Segre, C. (éd.) 2003. Textes littéraires français 968. Genève: Droz. 